\title{
3T MR-guided minimally-invasive penile fracture repair
}

\author{
Giovanni Rosi ${ }^{1^{*}}$, Paolo Fontanella ${ }^{2 *}$, Giordano Venzi ${ }^{2}$, Fernando Jermini ${ }^{2}$, Filippo Del Grande ${ }^{1}$ \\ ${ }^{1}$ Radiology Department, Ospedale Regionale di Lugano, Switzerland. \\ ${ }^{2}$ Urology Department, Ospedale Regionale di Lugano, Switzerland. \\ * These Authors equally contributed to the development of this paper.
}

\begin{abstract}
Summary We present the case of a 21 year old patient with an incomplete tear of the tunica albuginea occurred after violent masturbation. The diagnostic assessment was performed first clinically, then with ultrasound and with 3 Tesla MRI. 3 Tesla MRI, owing to its high resolution, allowed to exactly detect the tear location leading to precise preoperative planning. After adequate diagnosis through imaging and proper planning, we were able to perform a selective minimally invasive surgical approach to repair the lesion.
\end{abstract}

KEY WORDS: Penile fracture; Magnetic Resonance Imaging; Minimally invasive penile surgery.

Submitted 10.August.2015; Accepted 13.October 2015

\section{Case report}

A twenty-one year-old Caucasian male presented to our emergency department with an asymmetric penile swelling, penile-scrotal hematoma, and severe localized pain. The patient reported a cracking sound followed by sudden pain and rapid detumescence of the penis after violent masturbation 48 hours before presenting to the emergency room.

Clinical examination showed a painful and swollen penis, mainly on the right ventral side. Purple colored penile skin extended from the proximal penile shaft to right side of scrotum, without association of urethrorrhagia. On clinical examination no significant "gaps" were palpated. An ultrasound exam was performed to evaluate the integrity of the tunica albuginea and the extension of the hematoma.

The exam confirmed the presence of a large hematoma extending from the right central-third penile shaft to the region of the glans penis. No clear evidence of discontinuity of the tunica albuginea was detected. In order to plan the best surgical approach, the patient underwent a 3 Tesla magnetic resonance (MR) of the penis. The MR showed a tear in the proximal-third of the right ventral tunica albuginea with a large surrounding hematoma in the soft tissues of the right penile shaft. The hematoma appeared to be enclosed between the tunica albuginea, torn, and the overlying Buck's fascia, intact. MR findings allowed to approach the lesion directly, suture the sheath tear and drain the hematoma.

\section{Discussion}

Penile fracture is a rare urologic emergency characterized by the rupture of one or both corpora cavernosa, associated with the tear of tunica albuginea. It is difficult to estimate the incidence of penile fractures: a review of 1,600 cases reported in the literature, showed an higher prevalence in Asian and Middle-Eastern countries. Vigorous sexual intercourse is considered to be the main cause of penile fractures in the Western world. Other causes are the following: non-physiologic penile bending, rolling over in bed with an erect penis and sport injuries with flaccid penis. In Middle Eastern countries the most frequent reported cause of penile fracture is a self-inflicted practice called Taghaandan which consists of snapping and bending the erect penis to achieve a rapid detumescence (1). Similarly to our case, penile fracture occurs with an audible popping or cracking sound followed by intense pain and rapid detumescence of the broken corpus cavernosus, followed by a briefly delayed generalized penile detumescence. The common clinical presentation of a penile fracture is the so-called "eggplant deformity", (i.e.: asymmetrical swelling of the penis with purple bruises over the entire shaft possibly extending to the scrotum and perineum). Hematuria, urethrorrhagia and voiding difficulties are common signs of urethral involvement, which is estimated to be as high as $38 \%$ with a prevalence of $20 \%$ of urethral bleeding. In order to achieve adequate treatment planning, it is very important to differentiate false ruptures from real ones, as the latter requires surgical treatment. The false rupture is the presence of a hematoma confined in the soft tissues due to rupture of a superficial or deep dorsal vein and not to actual sheaths rupture. The intracavernous hematoma is considered false rupture and needs conservative treatment.

Ultrasound, cavernosography, retrograde urethrography and MR are the diagnostic imaging tools to assess penile fractures. Ultrasound is most commonly used for early diagnosis, to evaluate the integrity of the tunica albuginea, and the presence and dimensions of a hematoma. However, the low sensitivity for small lesions of the tunica albuginea and the high operator dependence are major limitations. Retrograde urethrography and cavernosography are rarely performed and have been generally replaced by MR. Today, MR represents the gold standard to show the extension and the exact location of the lesions of the 
tunica albuginea, the presence of an intra or extra-cavernous hematoma, and the possible involvement of the corpus spongiosum and the urethra (3). In an uninjured penis, MR doesn't allow to distinguish the tunica albuginea from Buck's fascia; only by isolate rupture of the tunica albuginea, an hematoma interposes between the two fascia, granting a vision of the two separate layers. Surgical treatment is mandatory in cases of rupture of the tunica albuginea and urethral involvement (3).

MR plays a key role for treatment planning. High resolution images permit to distinguish a real tunica albuginea rupture, from a false rupture due to a dorsal vein tear or an isolated intracavernous hematoma. In case of real rupture conservative approach often leads to penile curvature and erectile dysfunctions, urethral stricture, and infection of the hematoma (4).

In our patient, after detecting the location of the rupture with MR, we opted for a minimally-invasive ventral approach instead of a degloving procedure, to preserve the integrity of the foreskin, avoiding unnecessary circumcision, reducing the risk for necrotic and sensitivity complications.

The ventral approach was preferred as it's less traumatic and determines rapid healing, while granting ideal expo- sure of both the hematoma and the damaged area of tunica albuginea, allowing for proper repair (Figure 1).

\section{Conclusions}

Penile fracture is an uncommon condition that requires accurate diagnosis for treatment planning. High resolution MR imaging provides the key information to offer the best treatment minimizing risks of complications. With high resolution 3 Tesla MR imaging, we were able to properly plan and optimally treat, obtaining excellent functional and aesthetic result.

\section{References}

1. Zargooshi J. Penile fracture in Kermanshah, Iran: report of 172 cases. J Urol. 2000; 164:364-6.

2. Choi MH, Kim B, Ryu JA, et al. MR imaging of acute penile fracture. Radiographics. 2000; 20:1397-405. Erratum in: Radiographics. 2000; 20:1818.

3. Parker RA $3^{\text {rd }}$, Menias CO, Quazi R, et al. MR Imaging of the penis and scrotum. Radiographics. 2015; 35:1033-50.

4. Lumen N, Kuehhas FE, Djakovic N, et al. Review of the current management of lower urinary tract injuries by the EAU Trauma Guidelines Panel. Eur Urol. 2015; 67:925-9.

\section{Figure 1.}

A. Upon arrival at emergency department: markedly swollen, purple and painful penis with clinically suspected penile fracture.

B. Coronal fat suppressed T2 TSE (TR/TE 6000/120 ms) showing the interruption of the right tunica albuginea consistent with a tear (white arrow).

C. Intraoperative picture shows the rupture of the right tunica albuginea (black arrow), with a minimal-invasive surgical approach.

D. Post-surgical control after 14 days shows the penis with regular shape and appearance.

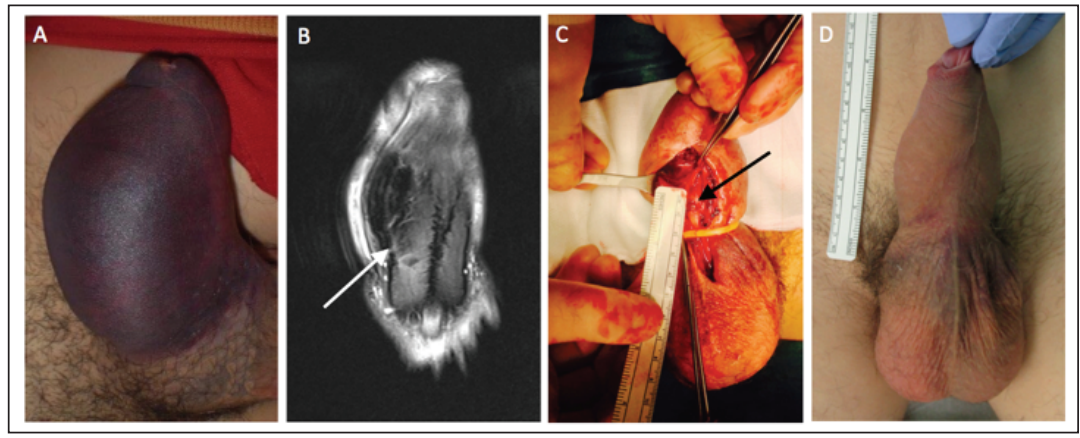

Correspondence

Giovanni Rosi, MD

Filippo Del Grande, MD

Ospedale Regionale di Lugano, Radiology Department

Via Tesserete 46, 6900 Lugano, Switzerland

Paolo Fontanella, MD (Corresponding Author)

p.fontanella@outlook.com

Giordano Venzi, MD

Fernando Jermini, MD

Ospedale Regionale di Lugano, Urology Department

Via Tesserete 46, 6900 Lugano, Switzerland 